\title{
The structure of the $\mathrm{C}_{4}$ cluster radical
}

\author{
J. R. Heath and R. J. Saykally \\ Department of Chemistry, University of California, Berkeley, California 94720
}

(Received 9 November 1990; accepted 10 December 1990)

Within the family of small carbon clusters, $\mathrm{C}_{4}$ has received a particularly notable amount of attention. ${ }^{1}$ Beginning with the extended Huckel calculations of Pitzer and Clementi in $1958,{ }^{2}$ many $a b$ initio calculations have addressed the structures and energetics of the two most stable isomers. $^{3-13}$ The most sophisticated calculations now agree that the bicyclic rhombus $\left({ }^{1} A_{g}\right)$ structure and the linear triplet $\left({ }^{3} \Sigma_{g}^{-}\right)$structures are approximately isoenergetic.

Prior to 1988, the only experimental information available for $\mathrm{C}_{4}$ consisted of an EPR and optical study of triplet $\mathrm{C}_{4}$ isolated in rare gas matrices. ${ }^{14}$ However, four important experiments have appeared in the past two years. Yang et $a l . .^{15}$ have measured the photoelectron spectrum of $\mathrm{C}_{4}^{-}$and have found evidence for the linear triplet isomer. Algranati et al., ${ }^{16}$ have reported the observation of the rhombic isomer in a Coulombic explosion experiment of photodetached $\mathrm{C}_{4}^{-}$. Shen and Graham ${ }^{17}$ reported a definitive assignment of the $v_{3}\left(\sigma_{u}\right)$ vibration of the triplet isomer in a matrix infrared experiment. The most surprising new result is that of Cheung and Graham, ${ }^{18}$ who presented evidence from new EPR measurements that the triplet state of $\mathrm{C}_{4}$ is actually slightly bent. This is not supported by any of the $a b$ initio calculations.

In this letter, we report the measurement of the $v_{3}\left(\sigma_{u}\right)$ fundamental vibration-rotation band of jet-cooled ${ }^{3} \Sigma_{g}-C_{4}$ through diode laser absorption spectroscopy. In this first high resolution study of the $\mathrm{C}_{4}$ cluster, we find no evidence for nonlinearity.

The diode laser apparatus used in recent studies of $\mathrm{C}_{5}$ (Ref. 19), $C_{7}$ (Refs. 20 and 21), and $C_{9}$ (Ref. 22) has been described in several recent publications. In brief, carbon clusters are generated by K.rF excimer laser ablation of a rotating, translating graphite rod placed in the throat of a pulsed, planar, argon jet. The expanding carrier gas/ carbon cluster mixture intersects 10 passes of a diode laser beam over a $15 \mu$ s time interval. The diode laser is focused onto a $\mathrm{HgCdTe}$ detector and the diode laser power is monitored by gated integration of the preamplified, filtered detector output. The diode laser is stepped in ca. $20 \mathrm{MHz}$ frequency intervals every $80-100$ shots, and the absorption signal is averaged over a 300 shot time constant. Immediately following the measurement of an absorption line, absolute and relative frequency calibrations are performed by measuring the absorption spectrum of $\mathrm{NH}_{3}$ or $\mathrm{H}_{2} \mathrm{O}$ and the fringe spectrum of an air-spaced, temperature-stabilized germanium etalon. The measurements have a frequency precision of ca. $0.002 \mathrm{~cm}^{-1}$.

Guided by the IR spectrum of matrix-isolated $\mathrm{C}_{4}$ reported by Shen and Graharn, ${ }^{17}$ much of the region between $1540-1560 \mathrm{~cm}^{-1}$ was searched. Twelve rovibrational lines assignable to a single vibrational band of a linear triplet molecule were observed. The calculated stick spectrum, along with typical data, are presented in Fig. 1. The frequencies and their respective assignments are presented in Table I. Derived spectroscopic constants and relevant $a b$ initio predictions are presented in Table II. The band was assigned to $\mathrm{C}_{4}$ based on the following: 1) the fitted spectroscopic constants are in excellent agreement with theory; 2 ) the measured band origin is in excellent agreement with both theory and matrix data (corrected for a matrix shift); 3 ) the chemistry of this absorber with respect to added impurities in the Ar carrier gas is consistent with that of other bare carbon clusters.

The lowest state of linear $\mathrm{C}_{4}$ is ${ }^{3} \Sigma_{g}^{-}$; consequently, except for the lowest $J$ states, each rovibrational transition should actually be a sum of three separate transitions corresponding to $J=N+1, N$, and $N-1$ states, where $N$ is the rotational quantum number, $J$ is the spin plus rotation quantum number, and 1 is the electron spin $(S)$ for a triplet molecule. The magnitude of the triplet splitting will be determined by the spin-rotation constant $(\gamma)$ and the spin-spin constant $(\lambda)$. The values of these terms have been measured by Graham et al. ${ }^{14}$ to be $0.0006(2) \mathrm{cm}^{-1}$ and $0.128(3) \mathrm{cm}^{-1}$, respectively. Assuming similar upper and lower state spin splitting constants, only the $R(2)$, $P(2)$ and $P(4)$ splittings are calculated to be greater than the resolution of our spectrometer (ca. $100 \mathrm{MHz}$ ). Only in the case of the $P(4)$ transition was our sensitivity sufficient to detect separated spin components, and this partially resolved transition is shown in Fig. 1. Although no components of either the $R(0)$ or $P(2)$ lines were observed, the measured spin splitting of the $P(4)$ line is sufficient for unambiguous assignment of the spectrum.

A further test of the open shell nature of this cluster was done by measuring the effect of a 100-200 G (inhomogeneous) magnetic field on the $P(6)$ absorption line. The $P(6)$ line was observed to broaden from a zero-field linewidth of $120 \mathrm{MHz}$ to about $200 \mathrm{MHz}$ in the presence of the field.

Since spin splittings were observed for only one rovibrational transition, the rotational constants $B^{\prime \prime}, B^{\prime}, D^{\prime}$, and the band origin $v_{3}$ were extracted by a least squares fit of the center frequency of the observed transitions to the energy expression

$$
E_{\mathrm{vr}}=(v+1 / 2) v_{3}+B_{v}(N(N+1))-D_{v}[N(N+1)]^{2} \text {. }
$$

The ground-state centrifugal distortion constant $D^{\prime \prime}$ was not statistically significant, and thus was fixed at 0.0 for the final fit. For the $P(4)$ line, splittings were calculated from the Schlapp expressions ${ }^{23}$ for a ${ }^{3} \Sigma$ molecule

$$
\begin{aligned}
E_{\mathrm{vrs}}(J & =N+1) \\
& =E_{\mathrm{vr}}-[2 \lambda(N+1) /(2 N+3)]+\gamma(N+1)
\end{aligned}
$$




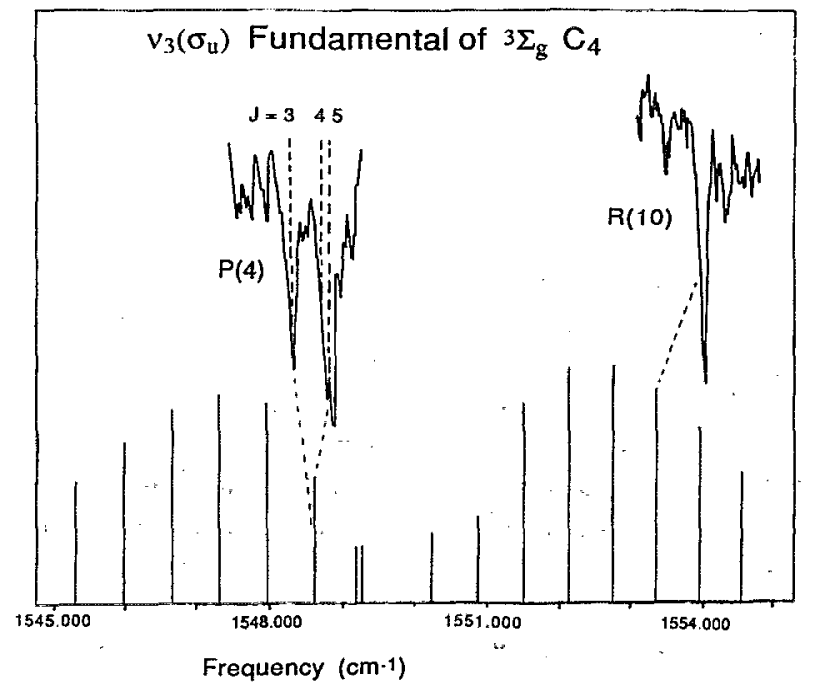

FIG. 1. Fitted rovibrational stick spectrum and experimental data of the $v_{3}\left(\sigma_{u}\right)$ antisymmetric stretch fundamental of the ${ }^{3} \Sigma_{g}$ isomer of $\mathrm{C}_{4}$. The rotational temperature of $\mathrm{C}_{4}$ is estimated to be near $30 \mathrm{~K}$.

$E_{\mathrm{vrs}}(J=N)=E_{\mathrm{vr}}$

$E_{\mathrm{vrs}}(J=N-1)=E_{\mathrm{vr}}-[2 \lambda N /(2 N-1)]-\gamma N$

with spin splitting parameters taken from Ref. 14. The deviations of the calculated from the observed transitions presented in Table I show a systematically larger error for low $N$. This is most likely due to a broadening of the low $N$ lines from unresolved spin multiplets.

\section{Is the triplet form of $\mathrm{C}_{4}$ linear?}

Cheung and Graham ${ }^{17}$ recently proposed that triplet $\mathrm{C}_{4}$ is actually slightly bent, at least when isolated in an argon matrix at $12 \cdot \mathrm{K}$. This conclusion was reached on the basis of resolving doublet structures in the EPR spectrum of ${ }^{12} \mathrm{C}_{4}$, which were interpreted as evidence of inequivalent $x$ and $y$ axes in the molecule. Experiments were conducted to test for inequivalent matrix sites and $\mathbf{H}$ atom interactions, and it was concluded that neither phenomena was the cause for the splitting.

TABLE I. Observed frequencies $\left(\mathrm{cm}^{-1}\right)$ assigned to the $v_{4}\left(\sigma_{u}\right)$ antisymmetric stretch fundamental of $\mathrm{C}_{4}$. The standard deviation of the fit was $0.00306 \mathrm{~cm}^{-1}$. Parenthetical values are calculated.

\begin{tabular}{lccccr}
\hline \hline$N$ & $R(N)$ & $\begin{array}{c}\text { obs-calc } \\
(x 10-3)\end{array}$ & $P(N)$ & $\begin{array}{r}\text { obs-calc } \\
(x 10-3)\end{array}$ \\
\hline 0 & $(1549.2665)$ & & & & \\
$2 J=2$ & 1549.9188 & -3.7 & $J=2$ & $(1548.2740)$ & \\
4 & 1550.5717 & -1.8 & $J=5$ & 1547.6108 & 1.4 \\
& & & $J=4$ & 1547.6108 & 4.1 \\
& & & $J=3$ & 1547.5993 & -0.1 \\
6 & 1551.2205 & 1.5 & & 1546.9375 & 3.1 \\
8 & 1551.8584 & 0.9 & & 1546.2524 & -3.9 \\
10 & 1552.4864 & -1.3 & & 1545.5704 & -1.1 \\
12 & 1553.1107 & 2.5 & & 1544.8791 & 0.6 \\
14 & 1553.7153 & -1.1 & & \\
\hline \hline
\end{tabular}

TABLE II. Measured and predicted molecular constants for the $v_{3}\left(\sigma_{u}\right)$ antisymmetric stretch fundamental of $\mathrm{C}_{4}$. Quoted uncertainties are $1 \sigma$.

\begin{tabular}{|c|c|c|}
\hline & Experiment & Theory \\
\hline$v_{3}$ & $1548.9368(21) \mathrm{cm}^{\cdots 1}$ & 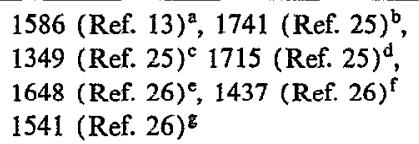 \\
\hline$B^{\prime \prime}$ & $0.16542(5) \mathrm{cm}^{-1}$ & 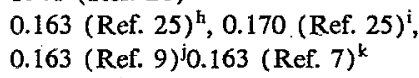 \\
\hline$B^{\prime}$ & $0.16486(7) \mathrm{cm}^{-1}$ & \\
\hline$D^{\prime}$ & $87(19) \mathrm{e}-08 \mathrm{~cm}^{-1}$ & \\
\hline$r_{\text {eff }}$ & $1.30431(21) \mathrm{A}$ & $\begin{array}{l}\left(1.308_{o}, 1.330_{m}(\text { Ref. } 25)^{\mathrm{h}}\right. \\
\left(1.283_{o} 1.305_{m}\right)(\operatorname{Ref} .25)^{\mathrm{i}} \\
\left(1.316_{o}, 1.297_{m}\right),(\operatorname{Ref} .9)^{\mathrm{j}} \\
\left(1.305_{o}, 1.300_{m}\right)(\operatorname{Ref} .7)^{\mathrm{k}}\end{array}$ \\
\hline
\end{tabular}

Levels of theory: (a) MP2/6-31G, (b) UHF/6-31G, (c) ROHF/4s2p1d, (d) $\mathrm{UHF} / 4 s 2 p 1 d$, (e) HF/4-21G, (f) MP2/4-21G, (g) ad hoc/MNDO, (h) SCF, ${ }^{(i)}$ D-MBPT (4), (j) SRCI, (k) HF/3-21G. o-Denotes outer C-C bond length. $m-$ Denotes middle $\mathrm{C}-\mathrm{C}$ bond length.

We find no evidence for a bent structure in the present study, and, indeed, the results presented here strongly support ab initio predictions of a linear, cumulenic cluster with a ${ }^{3} \Sigma_{g}^{-}$ground state. Although it is not possible to completely rule out a bent structure from these experiments, it is possible to place an upper limit on how bent the cluster can be. If $\mathrm{C}_{4}$ were bent by greater than about $6^{\circ}$, the $K=1 \leftarrow 1$ transition would originate less than $40 \mathrm{~cm}^{-1}$ above the ground state, resulting in a second set of $P_{\text {- }}$ and $R$-branch transitions slightly displaced from those reported in Table I. The strongest of these transitions would be observable with a signal to noise of at least 3 to 1 . Scans which were sufficiently thorough to detect such transitions were performed through the frequency region 1540-1560 $\mathrm{cm}^{-1}$, and no lines other than those listed in Table I were observed. It thus seems likely that the nonlinearity of triplet $\mathrm{C}_{4}$ reported by Cheung and Graham is due to the matrix environment. Cheung and Graham do point out that other triplet radicals, notably $\mathrm{SiCO}$ and $\mathrm{SiNN},{ }^{24}$ are bent in an argon matrix while the unperturbed, gas phase structure is linear.

\section{ACKNOWLEDGMENTS}

This work was supported by grants from the NASA Innovative Science Grants Program (Grant No. NAGW.1022) and by the ONR Cluster Program (Grant No. N004-90-J-1368). J.R.H. is supported by the NASA SETI foundation. We thank Dr. Jan Martin for sending us his results prior to publication. We would also like to thank Dr. Sherwood Chang and Dr. Jill Tartar for their continued interest in this project.

\footnotetext{
${ }^{1}$ For an excellent review on this subject, see W. Weltner, Jr: and R. J. Van Zee, Chem. Rey. 89, 1713 (1989).

${ }^{2}$ K. S. Pitzer and E. Clementi, J. Am. Chem. Soc. 81, 4477 (1959).

${ }^{3}$ E. Clementi, J. Am. Chem. Soc. 83, 4501 (1961).

${ }^{4}$ S. J. Strickler and K. S. Pitzer, in Molecular Orbitals in Chemistry, Physics, and Biology, edited by B. Pullman and O.-O. Lowdin (Academic, New York, 1964).

${ }^{5}$ R. Hoffman, Tetrahedron 22, 521 (1966).

${ }^{\circ}$ Z. Slanina and R. Zahradnik, J. Phys. Chem. 81, 2252 (1977).

${ }^{7}$ R. A. Whiteside, R. Krishnan, D. J. DeFrees, J. A. Pople, and P. v. R. Schleyer, Chem. Phys. Lett. 78, 538 (1981).
} 
${ }^{8}$ G. Pacchioni and J. Koutccky, J. Chem. Phys. 88, 1066 (1988).

${ }^{9}$ J. P. Ritchie, H. F. King, and W. S. Young, J. Chem. Phys. 85, 5175 (1986).

${ }^{10} \mathrm{~K}$. Raghavachari and J. S. BinkJey, J. Chem. Phys. 87, 2191 (1987).

${ }^{11}$ B. K. Rao, S. N. Khanna, and P. Jenna, Solid State Commun. 58, 53 (1986).

${ }^{12}$ D. E. Bernholdt, D. H. Magers, and R. J. Bartlett, J. Chem. Phys. 89, 3612 (1988).

${ }^{13}$ D. Michalska, H. Chojnacki, B. A. Hess, Jr., and L. J. Schaad, Chem. Phys. Lett. 141, 376 (1987).

${ }^{14}$ W. R. M. Graham, K. I. Dismuke, and W. Weltner, Jr., Astrophys. J. 204, 301 (1976).

${ }^{15}$ S. Yang, K. J. Taylor, M. J. Craycraft, J. Conceicao, C. L. Pettiette, O. Cheshnovsky, and R. E. Smalley, Chem. Phys. Lett. 144, 431 (1988).

${ }^{16}$ M. Algranati, H. Feldman, D. Kella, E. Malkin, E. Milklazky, R. Naaman, Z. Vager, and Z. Zajfman, J. Chem. Phys. 90, 4617 (1989).
${ }^{17}$ L. N. Shen and W. R. M. Graham, J. Chem. Phys. 91, 5115 (1989).

${ }^{18}$ H. M. Cheung and W. R. M. Graham, J. Chem. Phys. 91, 6664 (1989).

${ }^{19}$ J. R. Heath, A. L. Cooksy, M. H. W. Gruebele, C. A. Schmuttenmaer, and R. J. Saykally, Science 244, 565 (1989).

${ }^{20}$ J. R. Heath, R. A. Sheeks, A. L. Cooksy, and R. J. Saykally, Science 249, 895 (1990).

${ }^{21} \mathrm{~J}$. R. Heath and R. J. Saykally, J. Chem. Phys. (in press).

${ }^{22}$ J. R. Heath and R. J. Saykally, J. Chem. Phys 93, 8392 (1990).

${ }^{23}$ R. Schlapp, Phys. Rev. 51, 342 (1937).

${ }^{24}$ R. R. Lembke, R. F. Ferrante, and W. Weltner, Jr., J. Am. Chem. Soc. 99, 416 (1977).

${ }^{25}$ D. H. Magers, R. J. Harrison, and R. J. Bartlett, J. Chem. Phys. 87, 2191 (1987).

${ }^{26} \mathrm{~J}$; M. L. Martin, J. P. Francois, and R. Gijbels, J. Chem. Phys. (in press). 